\title{
Introduction to Numerical Computing
}

\section{Dhere $\mathbf{P}^{*}$}

Department of Mathematics, Bharati Vidyapeeth University, Sadashiv Peth, Pune, Maharashtra, India

\begin{abstract}
The main aim of this paper is to understand the information to numerical computing. In this paper we solve some examples of numerical computing. The numerical computational techniques are the technique by which mathematical problems are formulated and they can be solved with arithmetic operations. Those techniques are basically numerical methods. Numerical method supports the solution of almost every type of problem. The numerical methods are classified depending upon the type of the problem.
\end{abstract}

Keywords: Numerical computing; Roots of equation

\section{Introduction to Numerical Computing}

Numerical computing is an approach for solving complex mathematical problems using only simple arithmetic operations [1]. The approach involves formulation of mathematical models physical situations that can be solved with arithmetic operations [2]. It requires development, analysis and use of algorithms. Numerical computations invariably involve a large number of arithmetic calculations and, therefore, require fast and efficient computing devices [3]. The microelectronic revolution and the subsequent development of high, low cost personal computers have had a profound impact on the application of numerical computing methods to solve scientific problems [4].

\section{Numerical Methods are Classified Basically Depending upon the Type of Problem}

\section{Numerical methods to find roots of equations}

In many Engineering and Science application we come across some algebraic and transcendental equation [5],

Ex. Consider the equation:

$\mathrm{F}(\mathrm{x})=\mathrm{x}^{\wedge} 2-3 \mathrm{x}+2$

Such equation is satisfied at some specific value of $\mathrm{x}$. These values are called roots of the equation. The solution of this equation can be obtained with the help of numerical methods [6].

\section{System of Linear Algebric Equation}

$$
\begin{aligned}
& \text { Consider, } \\
& 2 x+3 y=7 \\
& 5 x+8 y=18
\end{aligned}
$$

The values of $\mathrm{x}$ and $\mathrm{y}$ in these equation can be obtained with help of numerical method [7].

Direct method become complex for no. of variables.

Numerical method are the best solution for large system. Such equation are generated in analysis of electronic ckts. Large structures etc. [8].

\section{Floating Point Numbers}

The term floating point is derived from fact that there is fixed no of digit before and after the decimal point i.e., the decimal point can float [9].
There are also representation in which number of digit before and after the decimal no is set called as fixed point representation [10].

In general, floating point representations are slower and less accurate. Than fixed point representation, but they can handled a large range of numbers [11].

\section{Distinguish Between Analog Computing and Digital Computing}

\section{Analog computing}

Analog refers to the principle of solving a problem by using a tool which operates in way analogues to the problem $[12,13]$. Many real life measurable quantities are analog in nature: time, temperature, pressure, and speed, for instance. The basic requirement in the application of analog computers is the writing down of differential equations describing the physical system of interest [14,15].

\section{Digital Computing}

A digital computer is a computing device that operates on inputs which are discrete in nature $[16,17]$. The input data are numbers that may represents numeral, letters, or other special symbols. Digital computers are more accurate than analog computers. Digital computers are widely used for many different applications and are often called general purpose computer [18-20].

\section{Characteristics of Numerical Computing}

1. Accuracy: Every method of numerical computing introduces errors. They may be either due to using an appropriate in pace of an exact mathematical procedure or due to inexact representation and manipulation of numbers in the computer. These errors affect the accuracy of the results.

2. Efficiency: One more consideration in choosing a numerical method for solution of a mathematical model is efficiency.

*Corresponding author: Dhere P, Department of Mathematics, Bharati Vidyapeeth University, Sadashiv Peth, Pune, Maharashtra, India, Tel: 02024407131; E-mail: purvaanuradhadhere2000@gmail.com

Received October 12, 2018; Accepted October 25, 2018; Published October 31 2018

Citation: Dhere P (2018) Introduction to Numerical Computing. J Appl Computat Math 7: 423. doi: 10.4172/2168-9679.1000423

Copyright: (c) 2018 Dhere P. This is an open-access article distributed under the terms of the Creative Commons Attribution License, which permits unrestricted use, distribution, and reproduction in any medium, provided the original author and source are credited. 
It means the amount of effort required by both human and computer to implement the method.

3. Numerical stability: Another problem introduced by some numerical computing method is that of numerical instability. Errors introduced into a computation, from whatever source, propagate in different ways. In some cases, these errors tend to grow exponentially, with disastrous computational results.

\section{What is Accuracy? How it is Affected during the Process of Computation}

Accuracy: Every method of numerical computing introduces errors. They may be either due to using an appropriate in pace of an exact mathematical procedure or due to inexact representation and manipulation of numbers in the computer. These error affect the accuracy of the results.

\section{Process of numerical computing}

1. Formulation of a mathematical model.

2. Construction of an appropriate numerical method.

3. Implementation of the method to obtain a solution.

4. Validation of the solution.

\section{New Trends in Numerical Computing}

1. In recent years, the increasing power of computer hardware has affected the approach of numerical computing in several ways.

2. It has forced scientists and engineers to search for algorithms that are computationally fast and efficient.

3. An important new trend is the construction of algorithms that are computationally fast and easy.

4. And important new trend is the construction of algorithm to take advantages of specialized computer hardware such as vector computer and parallel computer.

5. Another trend is the use of sophisticated interactive graphics, in which the user can view the results graphically and advice the computer, graphically, on how to proceed further.

\section{Add the Following}

$0.3879 \mathrm{E} 7$ and $0.813 \mathrm{E} 7$

$0.3879 \mathrm{E} 7$

+0.813 E7

Answer: 1.2009 E7

\section{Subtract the Following}

0.4189628 E5 and 0.23818 E5

0.4189628 E5

-0.23818 E5

Answer: 0.180828 E5

\section{Rolles Theorem}

It states that for any continuous differentiable function that has two equal values at two district points the function must have a point with satisfies the function where first derivative is zero.
> If $\mathrm{f}(\mathrm{x})$ is continuous in a interval $\mathrm{a}<=\mathrm{x}<=\mathrm{b}$

> If $\mathrm{f}(\mathrm{x})$ exist in the interval $\mathrm{a}, \mathrm{x}, \mathrm{b}$

$f(a)=f(b)=0$ then

There exist at least one value of $\mathrm{x}$ say as $\mathrm{C}$ such that

$\mathrm{f}(\mathrm{c})=0 \mathrm{a}<\mathrm{c}<\mathrm{b}$

e.g.,

$f(x)=x^{\wedge} 2-5 x+4$ and the interval is $[1,4]$

Answer: $f(x)=x^{\wedge} 2-5 x+4$

$f(x)=2 x-5$

$f(1)=1^{\wedge} 2-5(1)+4$

$=0$

$\mathrm{F}(4)=4 \wedge 2-5(4)+4$

$=0$

$F^{\prime}(X)=2 X-5$

$2 \mathrm{X}=5$

$\mathrm{X}=5 / 2$.

\section{Mean Value Theorem}

If a function $f(X)$ continuous in the closed interval [a,b] and differentiable in the open interval then there exist a point $C$ in the interval $9 \mathrm{a}, \mathrm{b} 0$ such that $\mathrm{f}^{\prime}(\mathrm{c})$ is equal to the function average rate of change over the $9 a, b)$ i.e. $f(x)$ is continuous

$f(x)=[a, b]$ and $f(x)$ exist $(a, b)$ then there will be a point $C$ which lies in the interval $(\mathrm{a}, \mathrm{b})$ such that

$$
f(c)=f(b)-f(a) / b-a, a, c, b \text {. }
$$

\section{Example}

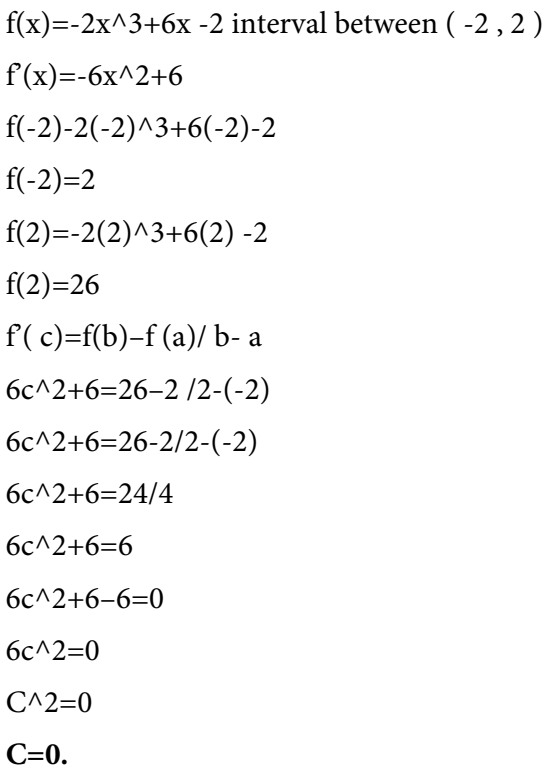

\section{Error and their Computation}

Whenever any mathematical calculation is performed it is not always possible to use exact and accurate values i.e., approximate values are used. 
Because of approximation the result is not accurate and we can say that error is introduced in the calculations.

\section{Types of Error}

\section{Absolute error}

It is defined as the magnitude of error. Which we considered as the true value. It is denoted by Ea.

\section{$\mathrm{Ea}=\mid$ Error $|=| \mathrm{Et} \mid$}

The absolute error depends upon the magnitude of actual and approximate value. Hence only absolute error does not provide complete information related to error.

\section{Relative error}

When the absolute error is normalized with respect to actual value then it is called relative error.

Relative error (er)=|absolute value-approximate value / true value $\mid$

$\%$ er=absolute error-approximate error/ true value ${ }^{\star} 100$

\section{Applications in Computing}

In an infinite series (e.g., $\log$ ) is approximated by a finite sum, the omitted term represents approximation error. Here, we need to estimate this error (or a bound for it) theoretically. Usually this error depends on some parameter: e.g., $\mathrm{n}=$ number of terms in the finite series $\mathrm{h}=$ "step size" in numerical differentiation/ integration in an interactive procedure we need to be able to test whether the process has converged.

\section{Conclusion}

We conclude that, Applications of numerical computing. Floating point and fixed points. Importance of numerical computing. Problems onarithmetic operation with floating point.

\section{References}

1. Bratu G (1914) On nonlinear integral equations. Bull Soc Math France 42: 191.

2. Jacobsen J, Shmitt K (2002) The Liouville-Bratu-Gelfand problem for radial operators. J Differential Equations 184: 283-298.

3. Buckmire R (2004) Applications of Mickens finite difference to several related boundary value problems. Advances in the Applications of Nonstandard Finite Difference Schemes 147: 47-87.

4. Caglar H, Caglar N, Ozer M, Valarstos A, Anagnostopoulos A (2010) B-spline method for solving Bratu's problem. Int J Compu Math 87: 1885-1891.

5. Zarebnia M, Sarvari Z (2012) Parametric spline method for solving Bratu's problem. Int J Non-linear Sci 14: 3-10.

6. Zarebnia M, Hoshyar M (2014) Solution of Bratu-type equation via spline method. Act Univ Apul 37: 61-72.

7. O Alayed, T Yuan, A Saaban (2016) Quintic spline method for solving linear and nonlinear boundary value problems. Sains Maly 45: 1007-1012.

8. Al-Towaiq M, Alayed O (2014) Ancient algorithm based on the cubic spline for the solution of Bratu-type equation. J Interdiscip Math 17: 471-484.

9. Abukhaled M, Khuri S, Sayfy A (2012) Spline-based numerical treatments of Bratu-type equations. Pales J Math 1: 63-70.

10. Rashidinia J, Maleknejad K, Taheri N (2013) Sinc-Galerkin method for numerical solution of the Bratus problems. Numer Algor 62: 1-11.

11. Zarebnia M, Sajjadian M (2014) Convergence of the Sinc-Galerkin method for the Bratu equation. Chiang Mai J Sci 41: 714-723.

12. Abbasbandy S, Hashemi M, Liu C (2011) The lie-group shooting method for solving the Bratu equation. Commun Nonlinear Sci Numer Simul 16: 42384249 .

13. Wazwaz A (2005) Decomposition method for a reliable treatment of the Bratutype equations. Appl Math Compu 166: 652-663.

14. Adesanya S, Babadipe E, Arekete S (2013) A new result on Adomian decomposition method for solving Bratus problem. Math Meth Model 3: 116 120

15. Vahidi, Hasanzade M (2012) Restarted Adomians decomposition method for the Bratu-type problem. Appl Math Sci 6: 479-486.

16. Deeba E, Khuri S, Xie S (2000) An algorithm for solving boundary value problems. J. Comput Phys 159 125-138.

17. Al-Mazmumy M, Al-Mutairi A, Al-Zahrani K (2017) Ancient decomposition method for solving Bratus boundary value problem. Amer J Compu Math 7 : 84-93.

18. Kashkari B, Abbas S (2017) Solution of initial value problem of Bratus type equation using modifications of Homotopy Perturbation method. Int $\mathrm{J}$ Comp Appl 162: 44-49.

19. Abolarin O (2013) New improved variational Homotopy Perturbation method for Bratu-type problems. Amer J Compu Math 3: 110-113.

20. Feng X, He Y, Meng J (2008) Applications of Homotopy Perturbation method to the Bratu-type equations. Topol Methods Nonlinear Anal 31: 243-252. 